\title{
On the approximation of the elastica functional in radial symmetry
}

Received: 26 July 2004 / Accepted: 19 October 2004

Published online: 22 December 2004 - (C) Springer-Verlag 2004

\begin{abstract}
We prove a result concerning the approximation of the elastica functional with a sequence of second order functionals, under radial symmetry assumptions. This theorem is strictly related to a conjecture of De Giorgi [8].
\end{abstract}

\section{Introduction}

Given a function $v \in H_{\mathrm{loc}}^{2}\left(\mathbb{R}^{n}\right)$ and a positive parameter $\varepsilon$ let us define

$$
\begin{gathered}
m_{\varepsilon}(v):=\frac{\varepsilon}{2}|\nabla v|^{2}+\varepsilon^{-1} W(v), \\
\operatorname{eul}_{\varepsilon}(v):=-\varepsilon \Delta v+\varepsilon^{-1} W^{\prime}(v),
\end{gathered}
$$

where $W: \mathbb{R} \rightarrow[0,+\infty[$ is a double well potential with two minima at \pm 1 , with $W(-1)=W(1)=0$, for instance $W(t)=\frac{1}{4}\left(1-t^{2}\right)^{2}$. Define the sequence $\mathcal{F}_{\varepsilon}: L_{\mathrm{loc}}^{1}\left(\mathbb{R}^{n}\right) \rightarrow[0,+\infty]$ of functionals as

$$
\mathcal{F}_{\varepsilon}(v):= \begin{cases}\int_{\mathbb{R}^{n}}\left[m_{\varepsilon}(v)+\varepsilon^{-1}\left(\operatorname{eul}_{\varepsilon}(v)\right)^{2}\right] d z & \text { if } v \in H_{\mathrm{loc}}^{2}\left(\mathbb{R}^{n}\right), \\ +\infty & \text { if } v \in L_{\mathrm{loc}}^{1}\left(\mathbb{R}^{n}\right) \backslash H_{\mathrm{loc}}^{2}\left(\mathbb{R}^{n}\right) .\end{cases}
$$

The aim of this paper is to begin to analyze the limit as $\varepsilon \rightarrow 0^{+}$, in the sense of $\Gamma-L_{\text {loc }}^{1}\left(\mathbb{R}^{n}\right)$-convergence, of the sequence $\left\{\mathcal{F}_{\varepsilon}\right\}$. Recall that

$$
M_{\varepsilon}(v):=\int_{\mathbb{R}^{n}} m_{\varepsilon}(v) d z
$$

G. Bellettini: Dipartimento di Matematica, Università di Roma "Tor Vergata", via della Ricerca Scientifica, 00133 Roma, Italy (e-mail: belletti@mat.uniroma2.it)

L. Mugnai: Dipartimento di Matematica, Università di Pisa, via Buonarroti 2, 56127 Pisa, Italy (e-mail: lmugnai@mail.dm.unipi.it)

Max Planck Institute for Gravitational Physics, Am Mühlenberg 1, 14476 Golm, Germany

The first author is grateful to Maurizio Paolini for useful discussions.

The second author gratefully acknowledges the hospitality and the support of the Max Planck Institute for Gravitational Physics in Golm, where this paper was completed. 
is the so-called Modica-Mortola (or Allen-Cahn) functional, whose $\Gamma-L_{\text {loc }}^{1}\left(\mathbb{R}^{n}\right)$ limit is a positive factor $c_{0}$ times the perimeter functional, see [9], [14], [7]. Notice also that $\operatorname{eul}_{\varepsilon}(v)$ (sometimes called chemical potential) is the $L^{2}$-gradient of $M_{\varepsilon}$ at $v$. It is well known that $\operatorname{eul}_{\varepsilon}(\cdot)$, if evaluated along solutions to the (possibly parabolic) Euler-Lagrange equation of $M_{\varepsilon}$, is strictly related to the mean curvature of the limit interface, see [12], [15], [11], [16]. We are precisely interested in analyzing the connections between $\operatorname{eul}_{\varepsilon}\left(v_{\varepsilon}\right)$ and the mean curvature of $\partial E$, when $\left\{v_{\varepsilon}\right\}$ is an arbitrary sequence of smooth functions approximating the characteristic function $\chi_{E}$ of the smooth set $E$, and having equibounded energy. Our interest in this question was originated by a conjecture made by De Giorgi concerning the $\Gamma$-limit of a sequence of functionals of the form $\mathcal{H}_{\varepsilon}(v):=\int_{\mathbb{R}^{n}}\left[1+\left(\operatorname{eul}_{\varepsilon}(v)\right)^{2}\right] m_{\varepsilon}(v) d z$, see [8] for a precise statement.

Observe that, by well known results concerning the asymptotic behaviour of $\left\{M_{\varepsilon}\right\}$, when $n=1$, the $\Gamma-L_{\text {loc }}^{1}(\mathbb{R})$-limits of $M_{\varepsilon}$ and of $\mathcal{F}_{\varepsilon}$ coincide. This shows, as expected, that in the one-dimensional case no curvature effect is present in the limit. Define, for $n=2$ and an open set $E \subset \mathbb{R}^{2}$ having smooth compact boundary $\partial E$,

$$
F(E):=\int_{\partial E}\left[1+\kappa^{2}\right] d \mathcal{H}^{1}
$$

where $\kappa$ is the curvature of $\partial E$ and $\mathcal{H}^{1}$ is the one-dimensional Hausdorff measure. $F$ is the so-called elastica functional, see for instance [3], [4].

For any $R>0$ set $B_{R}:=\left\{z \in \mathbb{R}^{2}:|z|<R\right\}$. Denote also by $\mu_{\varepsilon}^{v}$ the absolutely continuous measure

$$
\mu_{\varepsilon}^{v}(B):=\int_{B}\left[\varepsilon|\nabla v|^{2}+\varepsilon^{-1} W(v)\right] d z, \quad B \text { Borel set } \subseteq \mathbb{R}^{2} .
$$

Our main result is the following theorem.

Theorem 1.1. Let $n=2$ and let $\left\{v_{\varepsilon}\right\} \subset H_{\mathrm{loc}}^{2}\left(\mathbb{R}^{2}\right)$ be a sequence of radially symmetric functions such that

$$
\sup _{\varepsilon} \mathcal{F}_{\varepsilon}\left(v_{\varepsilon}\right)<+\infty
$$

If $\left\{v_{\varepsilon}\right\}$ converges to the characteristic function of a set $E$ in $L_{\text {loc }}^{1}\left(\mathbb{R}^{2}\right)$ and $\left\{\mu_{\varepsilon}\right\}$ weakly* converges to a Radon measure $\mu$, then the support of $\mu$ consists of a finite number of circles centered at the origin and contains $\partial E$. Moreover

$$
c_{0} F(E) \leq \liminf _{\varepsilon \rightarrow 0} \mathcal{F}_{\varepsilon}\left(v_{\varepsilon}\right) .
$$

It is clear that the radial symmetry assumption on $\left\{v_{\varepsilon}\right\}$ is a crucial simplification, and hence the above result is only a first step toward the understanding of the asymptotic behaviour of the sequences $\left\{\mathcal{F}_{\varepsilon}\right\}$ and $\left\{\mathcal{H}_{\varepsilon}\right\}$. Nevertheless, even in this simplified case, difficulties arise when trying to characterize the support of $\mu$ and in the proof of (6). The main obstruction is to derive sufficiently strong compactness properties of the sequence $\left\{v_{\varepsilon}\right\}$ from the assumption

$$
\sup M_{\varepsilon}\left(v_{\varepsilon}\right)<+\infty,
$$


and, more interestingly, from the uniform bound

$$
\sup _{\varepsilon} \varepsilon^{-1} \int_{\mathbb{R}^{2}}\left(-\varepsilon \Delta v_{\varepsilon}+\varepsilon^{-1} W^{\prime}\left(v_{\varepsilon}\right)\right)^{2} d z<+\infty .
$$

We stress that, even in the radially symmetric case, the proof of Theorem 1.1 reveals the importance of deriving a sufficiently strong control on the asymptotic behaviour of the so-called discrepancy functions (see (28) and (66) for the details). This is strictly related to proving that the measure $\mu$ has positive one-dimensional lower density.

Finally, observe that Theorem 1.1 is false in general, if we assume (7) but not (8). In addition, the constant $c_{0}$ is consistent with the bound given by the so-called $\Gamma$-limsup inequality (see [5]).

The content of the paper is the following. In Sect. 2 we introduce some notation. The main result of Sect. 3 is Corollary 3.5. Its proof is a consequence of Proposition 3.2, where a lower and upper estimate for the measure $\mu$ are established. The lower estimate relies on an adaptation of an inequality proved in [6], which in turn is based on assumption (8). Section 4 is devoted to the proof of Theorem 1.1, which follows from Theorem 4.1. The proof of Theorem 4.1 combines a blow-up argument centered at suitable points (where the whole sequence is far from the values \pm 1 ), which are rescaled back to the origin of the coordinates, and Corollary 3.5, in order to obtain a control of the associated sequence of discrepancy functions (see (28)). In Sect. 5 we make some comments and some generalizations to the radially symmetric case in higher space dimension.

\section{Notation}

For any $r>0$ set $B_{r}:=\left\{z \in \mathbb{R}^{2}:|z|<r\right\}$. For any $A \subseteq \mathbb{R}^{2}$, denote by $\chi_{A}$ the characteristic function of $A$, that is

$$
\chi_{A}(z):= \begin{cases}1 & \text { if } z \in A, \\ -1 & \text { if } z \notin A .\end{cases}
$$

Given $\rho>0$, we also let $T_{\rho}(A)$ be the set of all points of $\mathbb{R}^{2}$ whose distance from $A$ is less than $\rho$.

If $E \subset \mathbb{R}^{2}$ is an open set with compact boundary of class $\mathcal{C}^{2}$, we denote by $\kappa(z)$ the curvature of $\partial E$ at the point $z$ (positive for the circle). If we let

$$
d(z):=\operatorname{dist}\left(z, \mathbb{R}^{2} \backslash E\right)-\operatorname{dist}(z, E),
$$

the oriented distance function positive inside $E$, it is well known (see for instance [10]) that there exists $\rho>0$ such that $d$ is of class $\mathcal{C}^{2}$ in $T_{\rho}(\partial E)=\left\{z \in \mathbb{R}^{2}\right.$ : $|d(z)|<\rho\}$

$$
|\nabla d|=1 \quad \text { on } T_{\rho}(\partial E),
$$

and

$$
\frac{\kappa(\pi(z))}{1-d(z) \kappa(\pi(z))}=-\Delta d(z), \quad z \in T_{\rho}(\partial E),
$$


where $\pi(z):=z-d(z) \nabla d(z) \in \partial E$.

Let $R>0, \rho \in] 0, R\left[\right.$ and let us parametrize $\partial B_{R}$ using the arc length $s$. In the following we denote by $\left.\psi_{R}:\right]-\varrho, \varrho\left[\times\left[0,2 \pi R\left[\rightarrow T_{\varrho}\left(\partial B_{R}\right)\right.\right.\right.$ the map defined as

$$
\psi_{R}(d, s):=z, \quad z \in T_{\varrho}\left(\partial B_{R}\right), \quad d=d(z):=R-|z|, \quad s \in[0,2 \pi R[.
$$

If $J \psi_{R}$ denotes the jacobian of $\psi_{R}(d, s)=(R-d)(\cos (s / R), \sin (s / R))$, we have

$$
\left|\operatorname{det} J \psi_{R}(d, s)\right|=|1-d \kappa|, \quad \kappa:=1 / R .
$$

Given a radially symmetric function $u \in H_{\mathrm{loc}}^{1}\left(\mathbb{R}^{2}\right)$, we let $\left.\bar{u}:\right]-\rho, \rho[\times[0,2 \pi R[\rightarrow$ $\mathbb{R}$ be defined as $\bar{u}(d, s):=u\left(\psi_{R}(d, s)\right)=u(z)$. Since $u$ is radially symmetric, we have that $\bar{u}$ depends only on the variable $d$, i.e., $\bar{u}=\bar{u}(d)$. We denote by $\bar{u}^{\prime}$ the derivative of $\bar{u}$ with respect to $d$. Observe that

$$
\begin{aligned}
& \mu_{\varepsilon}^{u}\left(T_{\varrho}\left(\partial B_{R}\right)\right)=\int_{T_{\varrho}\left(\partial B_{R}\right)} m_{\varepsilon}(u) d z \\
= & 2 \pi R \int_{]-\varrho, \varrho[}\left[\frac{\varepsilon}{2}\left(\bar{u}^{\prime}(t)\right)^{2}+\varepsilon^{-1} W(\bar{u}(t))\right]|1-t \kappa| d t .
\end{aligned}
$$

Given $t \in]-\rho, \rho[$, we let

$$
\xi_{\varepsilon}^{\bar{u}}(t):=\frac{\varepsilon}{2}\left(\bar{u}^{\prime}(t)\right)^{2}-\frac{W(\bar{u}(t))}{\varepsilon}
$$

to be the discrepancy function associated with $\bar{u}$.

Let $w: \mathbb{R} \rightarrow \mathbb{R}$ be an even strictly convex smooth function such that $w^{-1}(0)=$ $\{ \pm 1\}$, negative inside] $-1,1$, positive outside, and satisfying $w^{\prime}(-1)<0, w^{\prime}(1)>$ 0 . Associated with $w$ we can define the cubic-like nonlinearity $f: \mathbb{R} \rightarrow \mathbb{R}$ as $f(\tau):=2 w(\tau) w^{\prime}(\tau)$, which has exactly the three zeros $-1,0,1$. We set $W(\tau):=$ $w(\tau)^{2}$. Then $W$ is the so-called double well potential, with two absolute minimizers \pm 1 at equal depth, and $W(-1)=W(1)=0$.

Given $W$ as above there exists a unique absolute minimizer $\gamma$ of the problem

$$
\begin{aligned}
\inf \left\{\int_{\mathbb{R}}\left(\frac{\left|\zeta^{\prime}\right|^{2}}{2}+W(\zeta)\right) d y\right. & : \zeta \in H_{\mathrm{loc}}^{1}(\mathbb{R}), \\
& \left.\zeta(0)=0, \lim _{y \rightarrow \pm \infty} \zeta(y)= \pm 1\right\} .
\end{aligned}
$$

The function $\gamma: \mathbb{R} \rightarrow]-1,1[$ is smooth, strictly increasing, and satisfies the equation

$$
-\gamma^{\prime \prime}+W^{\prime}(\gamma)=0
$$

Note that $\gamma^{\prime}=\sqrt{2 W(\gamma)}$. The constant $c_{0}$ in (6) is exactly the infimum in (14), and

$$
c_{0}=\int_{\mathbb{R}}\left(\frac{1}{2}\left|\gamma^{\prime}\right|^{2}+W(\gamma)\right) d y=\int_{\mathbb{R}}\left|\gamma^{\prime}\right|^{2} d y=\int_{]-1,1[} \sqrt{2 W(\tau)} d \tau .
$$


It is known that if a bounded function $\alpha \in H_{\mathrm{loc}}^{2}(\mathbb{R})$ satisfies (15) almost everywhere and is such that $0<\int_{\mathbb{R}}\left[\frac{\left|\alpha^{\prime}\right|^{2}}{2}+W(\alpha)\right] d y<+\infty$, then there exists $\tau \in \mathbb{R}$ such that either $\alpha(y)=\gamma(y+\tau)$ or $\alpha(y)=-\gamma(y+\tau)$. In other words, if $\alpha$ satisfies the Euler equation in (15) and has positive finite energy then it is a translated of $\gamma$ or of $-\gamma$.

If $J_{\varepsilon}$ are intervals contained in an interval $A \subseteq \mathbb{R}, f_{\varepsilon}: J_{\varepsilon} \rightarrow \mathbb{R}$ are functions, and if $J_{\varepsilon_{1}} \subset J_{\varepsilon_{2}}$ for $0<\varepsilon_{2}<\varepsilon_{1}$, and $J_{\varepsilon}$ invade $A$ as $\varepsilon \rightarrow 0^{+}$, we say that $\left\{f_{\varepsilon}\right\}$ converges to a function $f$ in $L_{\text {loc }}^{2}(A)$ as $\varepsilon \rightarrow 0^{+}$if given $\delta>0$ and a compact set $K \subset A$, there exists $\bar{\varepsilon}>0$ such that $\int_{K}\left|f_{\varepsilon}-f\right| d x<\delta$ for any $\left.\varepsilon \in\right] 0, \bar{\varepsilon}[$. We adopt a similar definition for convergence in $H_{\mathrm{loc}}^{k}(A), k=1,2$.

\section{Beginning of the proof of Theorem 1.1: density estimates}

In this section we establish the lower and upper density estimates for the measure $\mu$. Using these estimates we prove Corollary 3.5, which is a fundamental step in the proof of Theorem 4.1 in Sect. 4.

Let $\left\{v_{\varepsilon}\right\} \subset H_{\text {loc }}^{2}\left(\mathbb{R}^{2}\right)$ be a sequence satisfying (7). Here and in the following, by passing to a suitable subsequence, we can assume that there exists a Radon measure $\mu$ such that $\left\{\mu_{\varepsilon}^{v_{\varepsilon}}\right\}$ weakly* converges to $\mu$ as $\varepsilon \rightarrow 0^{+}$.

To prove the lower density estimate (ii) of Proposition 3.2 below, we need the following preliminary lemma (which is valid, with the same proof, in any space dimension $n$ ).

Lemma 3.1. Let $\left\{v_{\varepsilon}\right\} \subset H_{\mathrm{loc}}^{2}\left(\mathbb{R}^{2}\right)$ be a sequence satisfying (5). Let $\left.\eta_{0} \in\right] 0,1[$ be the minimum point for which $W^{\prime \prime} \geq 0$ on $] \eta_{0},+\infty\left[\right.$. Let $z_{0} \in \operatorname{spt}(\mu), r>0$ and $\eta \in\left[0, \eta_{0} / 2[\right.$. Then there exists $\bar{\varepsilon}>0$ such that

$$
\left.B_{r}\left(z_{0}\right) \cap\left\{\left|v_{\varepsilon}\right| \leq 1-\eta\right\} \neq \emptyset, \quad \varepsilon \in\right] 0, \bar{\varepsilon}[.
$$

Proof. We first establish the following inequality, which is a minor modification of [6, Lemma 4.4].

Let $A_{1}, A_{2}$ be two open subsets of $\mathbb{R}^{2}$ with $A_{1} \subset \subset A_{2} \subset \subset \mathbb{R}^{2}$. There exists a positive constant $C_{0}$ such that

$$
\begin{aligned}
& \int_{A_{1} \cap\{|u| \geq 1-\eta\}}\left[m_{\varepsilon}(u)+\varepsilon^{-1} W^{\prime 2}(u)\right] d z \\
\leq & C_{0} \eta \int_{A_{2} \cap\{|u| \leq 1-\eta\}} \varepsilon|\nabla u|^{2} d z+C_{0} \varepsilon \int_{\mathbb{R}^{2}}\left(\operatorname{eul}_{\varepsilon}(u)\right)^{2} d z \\
& +C_{0} \varepsilon^{1 / 2}\left[\int_{\mathbb{R}^{2}} m_{\varepsilon}(u) d z\right]^{1 / 2}
\end{aligned}
$$

for every $\eta \in\left[0, \eta_{0} / 2[\right.$, every $\left.\varepsilon \in] 0,1\right]$, and every $u \in H_{\text {loc }}^{2}\left(\mathbb{R}^{2}\right)$. 
Indeed, let $\phi \in \mathcal{C}_{c}^{\infty}\left(A_{2} ;[0,1]\right)$ be such that $\phi \equiv 1$ on $A_{1}$. Defining the function $g$ as in [6, Lemma 4.4] and arguing as in [6, formula (4.16)] we get

$$
\begin{aligned}
& \int_{A_{1} \cap\{|u| \geq 1-\eta\}}\left[\varepsilon W^{\prime \prime}(u)|\nabla u|^{2}+\frac{1}{2 \varepsilon} W^{\prime}(u) g(u)\right] d z \\
\leq & \frac{\varepsilon}{2} \int_{\mathbb{R}^{2}}\left(\operatorname{eul}_{\varepsilon}(u)\right)^{2} d z-\varepsilon \int_{A_{2} \cap\{|u| \leq 1-\eta\}} g^{\prime}(u)|\nabla u|^{2} d z \\
& +\varepsilon\left|\int_{A_{2}} g(u) \nabla u \cdot \nabla \phi d z\right| .
\end{aligned}
$$

Inequality (18) then follows reasoning as in [6] and observing that, using also the Hölder's inequality,

$$
\begin{aligned}
\left|\int_{A_{2}} \varepsilon g(u) \nabla u \cdot \nabla \phi d z\right| & \leq \varepsilon^{1 / 2}\|\nabla \phi\|_{\infty} \sup _{[-1,1]}|g|\left|A_{2} \backslash A_{1}\right|^{1 / 2}\left[\int_{\mathbb{R}^{2}} \varepsilon|\nabla u|^{2} d z\right]^{1 / 2} \\
& \leq C \varepsilon^{1 / 2}\left[\int_{\mathbb{R}^{2}} m_{\varepsilon}(u) d z\right]^{1 / 2} .
\end{aligned}
$$

We are now in a position to prove the lemma. Suppose by contradiction that there exists a decreasing sequence $\left\{\varepsilon_{j}\right\}$ of real numbers converging to zero such that $B_{r}\left(z_{0}\right) \subseteq\left\{\left|v_{\varepsilon_{j}}\right|>1-\eta\right\}$. Setting $A_{1}:=B_{r / 2}\left(z_{0}\right), A_{2}:=B_{r}\left(z_{0}\right)$ and applying (18) we get, using (5),

$$
\begin{aligned}
& 0<\mu\left(B_{r / 2}\left(z_{0}\right)\right) \leq \liminf _{j \rightarrow \infty} \mu_{\varepsilon_{j}}^{v_{\varepsilon_{j}}}\left(B_{r / 2}\left(z_{0}\right)\right) \\
& \leq \liminf _{j \rightarrow \infty} \int_{B_{r / 2}\left(z_{0}\right) \cap\left\{\left|v_{\varepsilon_{j}}\right|>1-\eta\right\}} m_{\varepsilon_{j}}\left(v_{\varepsilon_{j}}\right) d z \\
& \leq C_{0} \lim _{j \rightarrow \infty}\left[\varepsilon_{j} \int_{\mathbb{R}^{2}}\left(\operatorname{eul}_{\varepsilon_{j}}\left(v_{\varepsilon_{j}}\right)\right)^{2} d z+\varepsilon_{j}^{1 / 2}\left[\int_{\mathbb{R}^{2}} m_{\varepsilon_{j}}\left(v_{\varepsilon_{j}}\right) d z\right]^{1 / 2}\right]=0,
\end{aligned}
$$

which is a contradiction.

Proposition 3.2. Let $\left\{v_{\varepsilon}\right\} \subset H_{\mathrm{loc}}^{2}\left(\mathbb{R}^{2}\right)$ be a sequence of radially symmetric functions satisfying (7), and let $\mu$ be as above. The following estimates hold.

(i) There exists $C_{1}>0$ such that for every $z_{0} \in \operatorname{spt}(\mu) \backslash\{0\}$ there exists $\bar{r} \in] 0,\left|z_{0}\right|[$ such that

$$
\left.\mu\left(B_{r}\left(z_{0}\right)\right) \leq C_{1} r, \quad r \in\right] 0, \bar{r}[
$$

(ii) Assume in addition that (8) holds. Then there exists $C_{2}>0$ such that for every $z_{0} \in \operatorname{spt}(\mu) \backslash\{0\}$ there exists $\left.\bar{r} \in\right] 0,\left|z_{0}\right|[$ such that

$$
\left.\mu\left(B_{r}\left(z_{0}\right)\right) \geq C_{2} r, \quad r \in\right] 0, \bar{r}[.
$$


Proof. For every $r>0$ we set

$$
Q_{r}\left(z_{0}\right):=\left\{z \in \mathbb{R}^{2}:|| z|-| z_{0}||<r,\left|\arg (z)-\arg \left(z_{0}\right)\right|<r\right\},
$$

where $\arg (\cdot) \in] 0,2 \pi[$. Choose $r \in] 0,\left|z_{0}\right|\left[\right.$ and a sequence $\left.\left\{\left(\varepsilon_{i}, r_{i}\right)\right\} \subset\right] 0,+\infty\left[^{2}\right.$ such that $\lim _{i \rightarrow \infty} r_{i}=\lim _{i \rightarrow \infty} \varepsilon_{i} / r_{i}=0$, and

$$
\limsup _{r \rightarrow 0^{+}} \frac{\mu\left(Q_{r}\left(z_{0}\right)\right)}{r}=\lim _{i \rightarrow \infty} \frac{\mu_{\varepsilon_{i}}^{v_{\varepsilon_{i}}}\left(Q_{r_{i}}\left(z_{0}\right)\right)}{r_{i}} .
$$

Given a radially symmetric function $u \in H_{\text {loc }}^{2}\left(\mathbb{R}^{2}\right)$, we let $\left.\bar{u}:\right]-r, r[\times$ $\left[0,2 \pi\left|z_{0}\right|\left[\rightarrow \mathbb{R}\right.\right.$ be defined as $\bar{u}:=u \circ \psi_{\left|z_{0}\right|}$ (see (12)). Then, setting $\kappa:=1 /\left|z_{0}\right|$, we have

$$
\begin{gathered}
\quad \frac{1}{r_{i}} \int_{Q_{r_{i}}\left(z_{0}\right)} \frac{\varepsilon_{i}}{2}\left|\nabla v_{\varepsilon_{i}}\right|^{2} d z=2\left|z_{0}\right| \int_{]-r_{i}, r_{i}[} \frac{\varepsilon_{i}}{2}\left(\bar{v}_{\varepsilon_{i}}^{\prime}\right)^{2}|1-t \kappa| d t \\
\leq 2\left|z_{0}\right| \int_{]-r, r[} \frac{\varepsilon_{i}}{2}\left(\bar{v}_{\varepsilon_{i}}^{\prime}\right)^{2}|1-t \kappa| d t \leq \int_{T_{r}\left(\partial B_{\left|z_{0}\right|}\right)} m_{\varepsilon_{i}}\left(v_{\varepsilon_{i}}\right) d z \leq C
\end{gathered}
$$

and similarly

$$
\begin{aligned}
& \frac{1}{r_{i}} \int_{Q_{r_{i}}\left(z_{0}\right)} \frac{W\left(v_{\varepsilon_{i}}\right)}{\varepsilon_{i}} d z=2\left|z_{0}\right| \int_{]-r_{i}, r_{i}} \frac{W\left(\bar{v}_{\varepsilon_{i}}\right)}{\varepsilon_{i}}|1-t \kappa| d t \\
\leq & 2\left|z_{0}\right| \int_{]-r, r[} \frac{W\left(\bar{v}_{\varepsilon_{i}}\right)}{\varepsilon_{i}} d t \leq \int_{T_{r}\left(\partial B_{\left|z_{0}\right|}\right)} m_{\varepsilon_{i}}\left(v_{\varepsilon_{i}}\right) d z \leq C,
\end{aligned}
$$

where $C>0$ is the left hand side of (7). From (22) and (23), assertion (i) follows.

Let us now prove assertion (ii). Let $r \in] 0,\left|z_{0}\right|[$. By (17) and (5) we can select a decreasing sequence $\left\{\varepsilon_{i}\right\}$ converging to 0 as $i \rightarrow \infty$, such that

$$
B_{r / 2}\left(z_{0}\right) \cap\left\{\left|v_{\varepsilon_{i}}\right| \leq 1-\frac{\eta_{0}}{2}+\frac{1}{i}\right\} \neq \emptyset,
$$

for $i$ sufficiently large. By (7), we can also assume

$$
B_{r / 2}\left(z_{0}\right) \cap\left\{\left|v_{\varepsilon_{i}}\right| \geq 1-1 / i\right\} \neq \emptyset
$$

for $i$ large enough. By the radial symmetry of $v_{\varepsilon_{i}}$ it follows that there exist $r_{1}^{\varepsilon_{i}}, r_{2}^{\varepsilon_{i}}$ with $\left|z_{0}\right|-r / 2 \leq r_{j}^{\varepsilon_{i}} \leq\left|z_{0}\right|+r / 2$ for $j=1,2$, such that $\left|\bar{v}_{\varepsilon_{i}}\left(r_{1}^{\varepsilon_{i}}\right)\right| \leq 1-\frac{\eta_{0}}{2}+\frac{1}{i}$ and $\left|\bar{v}_{\varepsilon_{i}}\left(r_{2}^{\varepsilon_{i}}\right)\right| \geq 1-1 / i$. Reasoning as in [1, Lemma 1] we have

$$
\int_{] r_{1}^{\varepsilon_{i}}, r_{2}^{\varepsilon_{i}}[}\left[\frac{\varepsilon_{i}}{2}\left(\bar{v}_{\varepsilon_{i}}^{\prime}(t)\right)^{2}+\frac{W\left(\bar{v}_{\varepsilon_{i}}(t)\right)}{\varepsilon_{i}}\right] d t \geq \widehat{C}+O\left(\varepsilon_{i}\right),
$$

where $\widehat{C}>0$ is given by

$$
\begin{aligned}
\widehat{C}:=\inf \left\{\int_{] 0,+\infty[}\left(\frac{1}{2}\left|\zeta^{\prime}\right|^{2}+W(\zeta)\right) d y:\right. \\
\left.\quad \zeta \in H_{\mathrm{loc}}^{1}(] 0,+\infty[),|\zeta(0)|=1-\frac{\eta_{0}}{2}, \lim _{y \rightarrow+\infty}|\zeta(y)|=1\right\} .
\end{aligned}
$$




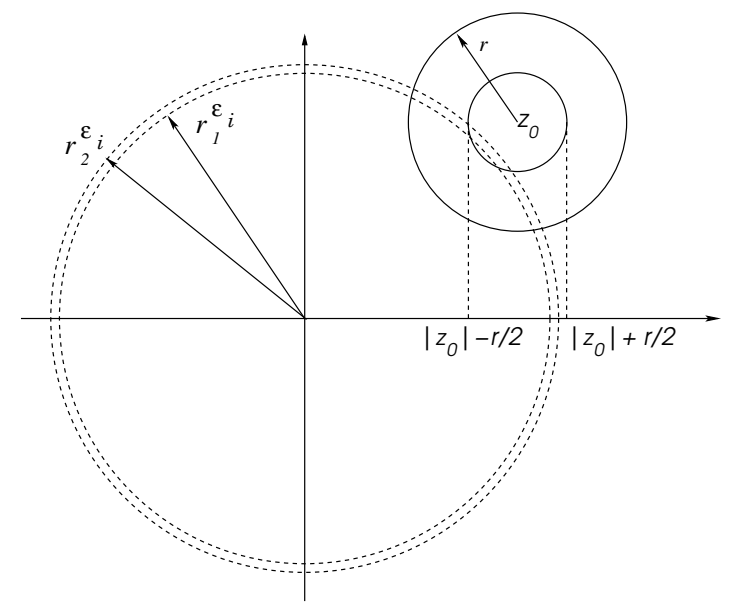

Fig. 1. Due to the choice of $r_{1}^{\varepsilon_{i}}, r_{2}^{\varepsilon_{i}}$, we have $\mathcal{H}^{1}\left(B_{r}\left(z_{0}\right) \cap \partial B_{r_{j}} \varepsilon_{i}\right) \geq r$ for every $r \in] 0,\left|z_{0}\right|[$, every $i \in \mathbb{N}$ and $j=1,2$

That is, a one-dimensional profile whose absolute value passes from two fixed values, one below $1-\frac{\eta_{0}}{2}+\frac{1}{i}$ and the other one above $1-1 / i$, pays at least a fixed positive amount of energy. Hence from (24) and using the radial symmetry of $v_{\varepsilon_{i}}$, we have

$$
C r+O\left(\varepsilon_{i}\right) \leq \mu_{\varepsilon_{i}}^{v_{\varepsilon_{i}}}\left(B_{r / 2}\left(z_{0}\right)\right) \leq \mu_{\varepsilon_{i}}^{v_{\varepsilon_{i}}}\left(B_{r}\left(z_{0}\right)\right)
$$

where $C>0$ does not depend on $\left|z_{0}\right|$, see Fig. 1. Letting $i \rightarrow+\infty$ in (25) assertion (ii) follows.

Remark 3.3. If each $v_{\varepsilon}$ is radially symmetric, then $\operatorname{spt}(\mu) \subset \mathbb{R}^{2}$ is radially symmetric, namely $z_{0} \in \operatorname{spt}(\mu)$ implies $\partial B_{\left|z_{0}\right|} \subset \operatorname{spt}(\mu)$. Indeed, using the notation in the proof of (22), if $z \in \partial B_{\left|z_{0}\right|}, \phi \in \mathcal{C}_{c}\left(B_{\rho}(z)\right)$, with $B_{\rho}(z) \subset T_{r}\left(\partial B_{\left|z_{0}\right|}\right)$, and if $\mathcal{R}$ denotes the rotation around the origin such that $\mathcal{R}(z)=z_{0}$, we have

$$
\begin{aligned}
& \int_{T_{r}\left(\partial B_{\left|z_{0}\right|}\right)} \phi(z) m_{\varepsilon}\left(v_{\varepsilon}\right) d z \\
= & \int_{] 0,2 \pi\left|z_{0}\right|[} \int_{]-r, r[} \phi(t, s)\left[\frac{\varepsilon}{2}\left(\bar{v}_{\varepsilon}^{\prime}(t)\right)^{2}+\frac{W\left(\bar{v}_{\varepsilon}(t)\right)}{\varepsilon}\right]\left(1-\frac{t}{\left|z_{0}\right|}\right) d t d s \\
= & \int_{] 0,2 \pi\left|z_{0}\right|[} \int_{]-r, r[} \phi\left(t, s-s_{0}\right)\left[\frac{\varepsilon}{2}\left(\bar{v}_{\varepsilon}^{\prime}(t)\right)^{2}+\frac{W\left(\bar{v}_{\varepsilon}(t)\right)}{\varepsilon}\right]\left(1-\frac{t}{\left|z_{0}\right|}\right) d t d s \\
= & \int_{T_{\varrho}\left(\partial B_{\left|z_{0}\right|}\right)} \phi(\mathcal{R} z) m_{\varepsilon}\left(v_{\varepsilon}\right) d z,
\end{aligned}
$$

where $s_{0}=\arg \left(z_{0}\right)\left|z_{0}\right|$ and $\psi_{\left|z_{0}\right|}\left(0, s_{0}\right)=z_{0}$. 
Remark 3.4. Assume that $\left\{v_{\varepsilon}\right\}$ converges in $L_{\text {loc }}^{1}\left(\mathbb{R}^{2}\right)$ to the characteristic function $\chi_{E}$ of a set $E \subset \mathbb{R}^{2}$. We observe that $\operatorname{spt}(\mu)$ contains the (reduced) boundary $\partial E$ of $E$. Indeed, let $z \in \partial E$, and $r>0$. Since $\mu_{\varepsilon}^{v_{\varepsilon}} \rightarrow \mu$ weakly* as $\varepsilon \rightarrow 0^{+}$, we have that for all $r$ such that $\mu\left(\partial B_{r}(z)\right)=0$ (in particular for almost every $r$ )

$$
\mu\left(B_{r}(z)\right)=\lim _{\varepsilon \rightarrow 0^{+}} \mu_{\varepsilon}^{v_{\varepsilon}}\left(B_{r}(z)\right) \geq c_{0} \mathcal{H}^{1}\left(B_{r}(z) \cap \partial E\right),
$$

where the last inequality follows by using the $\Gamma$-liminf inequality for $M_{\varepsilon}$. As a consequence we have $z \in \operatorname{spt}(\mu)$.

The following result is a first step toward the characterization of the support of $\mu$ in Theorem 1.1 .

Corollary 3.5. For any $\lambda>0, \operatorname{spt}(\mu) \cap\left(\mathbb{R}^{2} \backslash B_{\lambda}\right)$ is contained in a finite union of circles centered at the origin.

Proof. It is a consequence of $\mu\left(\mathbb{R}^{2}\right)<+\infty$, of (20), (21), the radial symmetry of $\operatorname{spt}(\mu)$ and [2, Theorem 2.5.6].

\section{Proof of Theorem 1.1}

We have observed in Remark 3.4 that $\operatorname{spt}(\mu)$ contains $\partial E$. In addition, for any $\lambda>0, \operatorname{spt}(\mu) \cap\left(\mathbb{R}^{2} \backslash B_{\lambda}\right)$ consists of a finite union of circles, see Corollary 3.5. In this section we prove in particular that $\operatorname{spt}(\mu)$ cannot contain a sequence of circles which accumulates at the origin.

Given an open set $A \subseteq \mathbb{R}^{2}$ and $u \in H_{\text {loc }}^{2}\left(\mathbb{R}^{2}\right)$ set

$$
\mathcal{F}_{\varepsilon}(u, A):=\int_{A}\left[m_{\varepsilon}(u)+\varepsilon^{-1}(\operatorname{eul}(u))^{2}\right] d z,
$$

and $\mathcal{F}_{\varepsilon}\left(u, \mathbb{R}^{2}\right):=\mathcal{F}_{\varepsilon}(u)$.

Theorem 4.1. Let $\left\{v_{\varepsilon}\right\} \subset H_{\mathrm{loc}}^{2}\left(\mathbb{R}^{2}\right)$ be a sequence of radially symmetric functions satisfying (5), and assume that $\left\{\mu_{\varepsilon}^{v_{\varepsilon}}\right\}$ weakly*-converges to a Radon measure $\mu$. Let $R>0$ be such that $\partial B_{R} \subseteq \operatorname{spt}(\mu)$. Then

$$
\liminf _{\varepsilon \rightarrow 0^{+}} \mathcal{F}_{\varepsilon}\left(v_{\varepsilon}, T_{\rho}\left(\partial B_{R}\right)\right) \geq 2 \pi c_{0}\left(R+\frac{1}{R}\right),
$$

for all $\rho \in] 0, R[$.

Proof. Possibly passing to a suitable subsequence (from now on all subsequences will not be relabelled) we can suppose that

$$
\sup _{\varepsilon} M_{\varepsilon}\left(v_{\varepsilon}\right)<+\infty, \quad \sup _{\varepsilon} \varepsilon^{-1} \int_{\mathbb{R}^{2}}\left(\operatorname{eul}_{\varepsilon}\left(v_{\varepsilon}\right)\right)^{2} d z<+\infty,
$$

that there exist $\lim _{\varepsilon \rightarrow 0} M_{\varepsilon}\left(v_{\varepsilon}\right)<+\infty, \lim _{\varepsilon \rightarrow 0} \varepsilon^{-1} \int_{\mathbb{R}^{2}}\left(\operatorname{eul}_{\varepsilon}\left(v_{\varepsilon}\right)\right)^{2} d z<+\infty$ and that $\left\{v_{\varepsilon}\right\}$ converges to a (finite perimeter) set $E$ in $L_{\text {loc }}^{1}\left(\mathbb{R}^{2}\right)$ as $\varepsilon \rightarrow 0^{+}$. 
Thanks to Corollary 3.5 , which implies that the circles composing spt $(\mu)$ cannot accumulate on $\partial B_{R}$, we select $\rho^{*}>0$ in such a way that a suitable asymptotic property on the discrepancy functions $\xi_{\varepsilon}^{v_{\varepsilon}}$ holds. Let us be more precise.

Step 1. There exists $\left.\rho^{*} \in\right] 0, R[$ such that

$$
\lim _{\varepsilon \rightarrow 0^{+}} \xi_{\varepsilon}^{\bar{v}_{\varepsilon}}(\rho)=\lim _{\varepsilon \rightarrow 0^{+}} \xi_{\varepsilon}^{\bar{v}_{\varepsilon}}(-\rho)=0
$$

for almost every $\rho \in] 0, \rho^{*}[$.

From Corollary 3.5 we can select $\lambda>0$ such that $\operatorname{spt}(\mu) \cap T_{\lambda}\left(\partial B_{R}\right)=\partial B_{R}$. Hence, given any $\tau \in] 0, \lambda / 2[$,

$$
\lim \mu_{\varepsilon}^{v_{\varepsilon}}\left(T_{\tau}\left(\partial B_{R-\frac{\lambda}{2}}\right)\right)=\lim \mu_{\varepsilon}^{v_{\varepsilon}}\left(T_{\tau}\left(\partial B_{R+\frac{\lambda}{2}}\right)\right)=0 .
$$

Using (13), it follows that there exists a subsequence such that

$$
\lim _{\varepsilon \rightarrow 0^{+}}\left[\frac{\varepsilon}{2}\left(\bar{v}_{\varepsilon}^{\prime}(t)\right)^{2}+\varepsilon^{-1} W\left(\bar{v}_{\varepsilon}(t)\right)\right]=0
$$

for almost every $t \in] R-\frac{\lambda}{2}-\tau, R-\frac{\lambda}{2}+\tau[\cup] R+\frac{\lambda}{2}-\tau, R+\frac{\lambda}{2}+\tau[$, and this provides the existence of $\rho^{*}$ in step 1 .

Observe that, since from Corollary 3.5, the set $\operatorname{spt}(\mu)$ consists of at most a countable set of circles which can accumulate only at the origin, assertion (28) actually holds for almost every $\rho \in] 0, R[$.

Possibly reducing $\rho^{*}$, we can assume that

$$
\left.\operatorname{det} J \psi_{R}(d, s) \in\right] 1 / 2,2[, \quad(d, s) \in]-\varrho, \varrho[\times[0,2 \pi R[.
$$

Fix now $\rho \in] 0, \rho^{*}[$ as in step 1 . It is well known [14] that

$$
2 \pi c_{0} R=c_{0} \mathcal{H}^{1}\left(\partial B_{R}\right) \leq \liminf _{\varepsilon \rightarrow 0} \mu_{\varepsilon}^{v_{\varepsilon}}\left(T_{\rho}\left(\partial B_{R}\right)\right) .
$$

To prove (26) it will be sufficient to show that

$$
\frac{2 \pi c_{0}}{R}=c_{0} \int_{\partial B_{R}}(\kappa(z))^{2} d \mathcal{H}^{1}(z) \leq \liminf _{\varepsilon \rightarrow 0} \varepsilon^{-1} \int_{T_{\rho}\left(\partial B_{R}\right)}\left(\operatorname{eul}_{\varepsilon}\left(v_{\varepsilon}\right)\right)^{2} d z .
$$

Indeed using (31) and (32) it follows

$$
\begin{aligned}
c_{0} \int_{\partial B_{R}}\left[1+\kappa^{2}\right] d \mathcal{H}^{1} \leq & \liminf _{\varepsilon \rightarrow 0} \mu_{\varepsilon}^{v_{\varepsilon}}\left(T_{\rho}\left(\partial B_{R}\right)\right) \\
& +\liminf _{\varepsilon \rightarrow 0} \varepsilon^{-1} \int_{T_{\rho}\left(\partial B_{R}\right)}\left(\operatorname{eul}_{\varepsilon}\left(v_{\varepsilon}\right)\right)^{2} d z \\
\leq & \liminf _{\varepsilon \rightarrow 0} \mathcal{F}_{\varepsilon}\left(v_{\varepsilon}, T_{\rho}\left(\partial B_{R}\right)\right),
\end{aligned}
$$

and (26) is proved.

Step 2. Construction of the blow-up sequence $\left\{V_{\varepsilon}\right\}$ around $\partial B_{R}$. 
For any $\varepsilon>0$ let $\left.I_{\varepsilon}:=\right]-\frac{\rho}{\varepsilon}, \frac{\rho}{\varepsilon}\left[\right.$, let $\phi_{\varepsilon}: I_{\varepsilon} \times[0,2 \pi R[\rightarrow]-\varrho, \varrho[\times[0,2 \pi R[$ be the map defined by

$$
\phi_{\varepsilon}(y, s):=(\varepsilon y, s)=(d, s), \quad(y, s) \in I_{\varepsilon} \times[0,2 \pi R[,
$$

and let us denote by $V_{\varepsilon}: I_{\varepsilon} \times[0,2 \pi R[\rightarrow \mathbb{R}$ the function

$$
V_{\varepsilon}:=\bar{v}_{\varepsilon} \circ \phi_{\varepsilon}
$$

By definition we have

$$
v_{\varepsilon}(z)=V_{\varepsilon}\left(\varepsilon^{-1} d, s\right)=V_{\varepsilon}(y, s), \quad z \in T_{\varrho}\left(\partial B_{R}\right),(y, s) \in I_{\varepsilon} \times[0,2 \pi R[.
$$

By assumption $v_{\varepsilon}$ is radially symmetric, hence $V_{\varepsilon}$ depends only on the variable $y$; we denote by $V_{\varepsilon}^{\prime}$ the derivative of $V_{\varepsilon}$ with respect to $y$.

By differentiating (33) with respect to $z$ we get $\nabla v_{\varepsilon}=\varepsilon^{-1} V_{\varepsilon}^{\prime} \nabla d$, hence $\left|\nabla v_{\varepsilon}\right|^{2}=\varepsilon^{-2}\left(V_{\varepsilon}^{\prime}\right)^{2}$, and from (11)

$$
\Delta v_{\varepsilon}=\varepsilon^{-2} V_{\varepsilon}^{\prime \prime}-\frac{\varepsilon^{-1} \kappa}{1-\varepsilon y \kappa} V_{\varepsilon}^{\prime}
$$

where $v_{\varepsilon}$ is evaluated at $z$ and $V_{\varepsilon}$ at the corresponding $y$. It follows that

$$
\operatorname{eul}_{\varepsilon}\left(v_{\varepsilon}\right)=\varepsilon^{-1} \sigma_{V_{\varepsilon}}+\frac{\kappa}{1-\varepsilon y \kappa} V_{\varepsilon}^{\prime}
$$

where

$$
\sigma_{V_{\varepsilon}}(y):=-V_{\varepsilon}^{\prime \prime}(y)+W^{\prime}\left(V_{\varepsilon}(y)\right), \quad y \in I_{\varepsilon} .
$$

Moreover from (13) we have

$$
\int_{T_{\varrho}\left(\partial B_{R}\right)} m_{\varepsilon}\left(v_{\varepsilon}\right) d z=2 \pi R \int_{I_{\varepsilon}}\left[\frac{\left(V_{\varepsilon}^{\prime}\right)^{2}}{2}+W\left(V_{\varepsilon}\right)\right]|1-\varepsilon y \kappa| d y
$$

and

$$
\begin{aligned}
& \varepsilon^{-1} \int_{T_{\varrho}\left(\partial B_{R}\right)}\left(\operatorname{eul}_{\varepsilon}\left(v_{\varepsilon}\right)\right)^{2} d z \\
= & 2 \pi R \int_{I_{\varepsilon}}\left[\varepsilon^{-1} \sigma_{V_{\varepsilon}}(y)+\frac{\kappa}{1-\varepsilon y \kappa} V_{\varepsilon}^{\prime}(y)\right]^{2}|1-\varepsilon y \kappa| d y .
\end{aligned}
$$

For clarity in the exposition, we will distinguish two cases:

Case 1. $\partial B_{R} \subseteq \partial E$;

Case 2. $\partial B_{R} \subseteq \operatorname{spt}(\mu) \backslash \partial E$. 
In Case 1, possibly passing to a subsequence, we can choose two sequences of real numbers $\left.\left\{a_{\varepsilon}\right\} \subset\right] 0, \rho\left[,\left\{b_{\varepsilon}\right\} \subset\right]-\rho, 0[$, such that

$$
\lim _{\varepsilon \rightarrow 0^{+}} a_{\varepsilon}=\lim _{\varepsilon \rightarrow 0^{+}} b_{\varepsilon}=0,
$$

and either

$$
\lim _{\varepsilon \rightarrow 0^{+}} \bar{v}_{\varepsilon}\left(a_{\varepsilon}\right)=-1, \quad \lim _{\varepsilon \rightarrow 0^{+}} \bar{v}_{\varepsilon}\left(b_{\varepsilon}\right)=1
$$

or

$$
\lim _{\varepsilon \rightarrow 0^{+}} \bar{v}_{\varepsilon}\left(a_{\varepsilon}\right)=1, \quad \lim _{\varepsilon \rightarrow 0^{+}} \bar{v}_{\varepsilon}\left(b_{\varepsilon}\right)=-1 .
$$

We will assume the validity of (39), the proof of (40) being similar.

The continuity of $\bar{v}_{\varepsilon}$ yields the existence of a sequence $\left.\left\{\delta_{\varepsilon}\right\} \subset\right] a_{\varepsilon}, b_{\varepsilon}[$ such that

$$
\lim _{\varepsilon \rightarrow 0^{+}} \delta_{\varepsilon}=0
$$

and

$$
\bar{v}_{\varepsilon}\left(\delta_{\varepsilon}\right)=0, \quad \varepsilon>0
$$

Possibly passing to a further subsequence, we can assume that all $\delta_{\varepsilon}$ have the same sign; without loss of generality, we assume that $\delta_{\varepsilon} \geq 0$ for any $\varepsilon$.

In Case 2, let us fix $\eta \in] 0, \eta_{0} / 2[$. Using (17) and the first estimate in (27), it is possible to find a sequence $\left.\left\{c_{\varepsilon}\right\} \subset\right]-\rho, \rho$ [ such that

$$
\lim _{\varepsilon \rightarrow 0^{+}} c_{\varepsilon}=0
$$

such that

$$
\left|\bar{v}_{\varepsilon}\left(c_{\varepsilon}\right)\right|=1-\eta, \quad \varepsilon>0
$$

Possibly passing to a subsequence, we can assume that $\bar{v}_{\varepsilon}\left(c_{\varepsilon}\right)$ has the same sign and similarly for $c_{\varepsilon}$; without loss of generality, we suppose

$$
\bar{v}_{\varepsilon}\left(c_{\varepsilon}\right)=1-\eta, \quad c_{\varepsilon} \geq 0 \quad \forall \varepsilon .
$$

Step 3. Compactness properties of $\left\{V_{\varepsilon}\right\}$.

Observe that there exists a constant $c>0$ such that

$$
V_{\varepsilon}(y) \in[-c, c], \quad \varepsilon>0, y \in I_{\varepsilon} .
$$

In fact, using (36), (30) and Young's inequality we get

$$
M_{\varepsilon}\left(v_{\varepsilon}\right) \geq 2 \pi R \int_{I_{\varepsilon}}\left[\frac{\left(V_{\varepsilon}^{\prime}\right)^{2}}{2}+W\left(V_{\varepsilon}\right)\right]|1-\varepsilon y \kappa| d y \geq \sqrt{2} \pi R \int_{I_{\varepsilon}}\left|V_{\varepsilon}^{\prime}\right| \sqrt{W\left(V_{\varepsilon}^{\prime}\right)} d y .
$$


Let $s_{\varepsilon}, t_{\varepsilon} \in \bar{I}_{\varepsilon}$ be such that $V_{\varepsilon}\left(s_{\varepsilon}\right)=\inf _{I_{\varepsilon}} V_{\varepsilon}$ and $V_{\varepsilon}\left(t_{\varepsilon}\right)=\sup _{I_{\varepsilon}} V_{\varepsilon}$. Then

$$
M_{\varepsilon}\left(v_{\varepsilon}\right) \geq \sqrt{2} \pi R \int_{] V_{\varepsilon}\left(s_{\varepsilon}\right), V_{\varepsilon}\left(t_{\varepsilon}\right)[} \sqrt{W(\tau)} d \tau .
$$

Then, if (46) does not hold, using (42) (or (45)) and (47) we get a contradiction with the first inequality in (27).

Using (46) we get that there exist $\alpha \in L_{\text {loc }}^{2}(\mathbb{R}) \cap L^{\infty}(\mathbb{R})$ such that, up to subsequences, $V_{\varepsilon} \rightarrow \alpha$ weakly in $L_{\text {loc }}^{2}(\mathbb{R})$ as $\varepsilon \rightarrow 0$. From (30) we get

$$
\pi R \int_{I_{\varepsilon}} \frac{\left(V_{\varepsilon}^{\prime}\right)^{2}}{2} d y \leq \pi R \int_{I_{\varepsilon}}\left(V_{\varepsilon}^{\prime}\right)^{2}|1-\varepsilon y \kappa| d y=\int_{T_{\varrho}\left(\partial B_{R}\right)} \frac{\varepsilon}{2}\left|\nabla v_{\varepsilon}\right|^{2} d z \leq M_{\varepsilon}\left(v_{\varepsilon}\right) .
$$

Hence, from the first inequality of (27) we have

$$
\sup _{\varepsilon}\left\|V_{\varepsilon}^{\prime}\right\|_{L^{2}\left(I_{\varepsilon}\right)}<+\infty .
$$

It follows that $\alpha \in H_{\mathrm{loc}}^{1}(\mathbb{R})$ and $V_{\varepsilon} \rightarrow \alpha$ weakly in $H_{\mathrm{loc}}^{1}(\mathbb{R})$ as $\varepsilon \rightarrow 0^{+}$. Observe that

$$
\int_{\mathbb{R}}\left(\frac{\left(\alpha^{\prime}\right)^{2}}{2}+W(\alpha)\right) d y<+\infty
$$

in particular $\alpha$ is not identically zero and $\alpha^{\prime} \in L^{2}(\mathbb{R})$. Indeed, if $K \subset \mathbb{R}$ is a compact set, from (36) and (30) we have

$$
M_{\varepsilon}\left(v_{\varepsilon}\right) \geq \pi R \int_{I_{\varepsilon} \cap K}\left(\frac{\left(V_{\varepsilon}^{\prime}\right)^{2}}{2}+W\left(V_{\varepsilon}\right)\right) d y
$$

so that

$$
+\infty>\lim _{\varepsilon} M_{\varepsilon}\left(v_{\varepsilon}\right) \geq \pi R \int_{K}\left(\frac{\left(\alpha^{\prime}\right)^{2}}{2}+W(\alpha)\right) d y
$$

and (49) follows by taking the supremum with respect to $K$ in (50).

Note that

$$
\begin{aligned}
\pi R \int_{I_{\varepsilon}} W\left(V_{\varepsilon}\right) d y & \leq 2 \pi R \int_{I_{\varepsilon}} W\left(V_{\varepsilon}\right)|1-\varepsilon y \kappa| d y \\
& =\varepsilon^{-1} \int_{T_{\varrho}\left(\partial B_{R}\right)} W\left(v_{\varepsilon}\right) d z \leq M_{\varepsilon}\left(v_{\varepsilon}\right) .
\end{aligned}
$$

Hence, recalling our assumptions on $W=w^{2}$, we get

$$
\sup _{\varepsilon}\left\|w\left(V_{\varepsilon}\right)\right\|_{L^{2}\left(I_{\varepsilon}\right)}<+\infty .
$$

Using (46) and (51) it follows

$$
\sup _{\varepsilon}\left\|W^{\prime}\left(V_{\varepsilon}\right)\right\|_{L^{2}\left(I_{\varepsilon}\right)} \leq 2 \max _{[-c, c]}\left|w^{\prime}\right| \sup _{\varepsilon}\left\|w\left(V_{\varepsilon}\right)\right\|_{L^{2}\left(I_{\varepsilon}\right)}<+\infty .
$$


By (34), using the second inequality of (27) and (48), by difference we get

$$
\sup _{\varepsilon} \varepsilon^{-1}\left\|\sigma_{V_{\varepsilon}}\right\|_{L^{2}\left(I_{\varepsilon}\right)}<+\infty \text {. }
$$

In particular

$$
\lim _{\varepsilon \rightarrow 0}\left\|\sigma_{V_{\varepsilon}}\right\|_{L^{2}\left(I_{\varepsilon}\right)}=0 .
$$

Hence, from (48) and (52) and recalling the expression (35) of $\sigma_{V_{\varepsilon}}$ we deduce

$$
\sup _{\varepsilon}\left\|V_{\varepsilon}^{\prime \prime}\right\|_{L^{2}\left(I_{\varepsilon}\right)}<+\infty .
$$

It follows that $\alpha \in H_{\text {loc }}^{2}(\mathbb{R})$ and

$$
V_{\varepsilon} \rightarrow \alpha \text { weakly in } H_{\text {loc }}^{2}(\mathbb{R}) \text { as } \varepsilon \rightarrow 0 .
$$

In particular, $V_{\varepsilon} \rightarrow \alpha, V_{\varepsilon}^{\prime} \rightarrow \alpha^{\prime}$ strongly in $L_{\text {loc }}^{2}(\mathbb{R})$ and $V_{\varepsilon} \rightarrow \alpha$ uniformly on the compact subsets of $\mathbb{R}$.

Step 4. The function $\alpha \in H_{\text {loc }}^{2}(\mathbb{R})$ obtained in step 3 is of class $\mathcal{C}^{2}$ and satisfies

$$
-\alpha^{\prime \prime}(t)+W^{\prime}(\alpha(t))=0, \quad t \in \mathbb{R} .
$$

Indeed, for any $\phi \in \mathcal{C}_{c}^{\infty}(\mathbb{R})$, using (56) we have

$$
\begin{aligned}
& \int_{\mathbb{R}}\left[-\alpha^{\prime \prime}+W^{\prime}(\alpha)\right] \phi d t=\int_{\operatorname{spt}(\phi)}\left[-\alpha^{\prime \prime}+W^{\prime}(\alpha)\right] \phi d t= \\
& \lim _{\varepsilon \rightarrow 0} \int_{\operatorname{spt}(\phi)}\left[-V_{\varepsilon}^{\prime \prime}+W^{\prime}\left(V_{\varepsilon}\right)\right] \phi d t \leq\|\phi\|_{\infty}|\operatorname{spt}(\phi)|^{1 / 2} \lim _{\varepsilon \rightarrow 0}\left\|\sigma_{V_{\varepsilon}}\right\|_{L^{2}\left(I_{\varepsilon}\right)}=0,
\end{aligned}
$$

where the last equality follows from (54). Hence (56) is satisfied almost everywhere, and by regularity everywhere.

Before passing to the next steps let us make the following important observation. From the previous computations, it follows that $\alpha$ is not identically zero, but we have not excluded, in general, that $\alpha$ is identically 1 or identically -1 (see Figure 2). Hence, we need a further step, which consists in fixing a point where all blow-up functions are far from \pm 1 . We reason as follows.

Step 5. Construction of the translated sequence $\left\{u_{\varepsilon}\right\}$ and its corresponding $\left\{U_{\varepsilon}\right\}$.

For any $\varepsilon>0$ define $u_{\varepsilon}$ as

$$
\begin{array}{ll}
u_{\varepsilon}(z):=\bar{v}_{\varepsilon}\left(R-|z|+\delta_{\varepsilon}\right), & z \in T_{\rho}\left(\partial B_{R+\delta_{\varepsilon}}\right) \quad \text { in Case 1, } \\
u_{\varepsilon}(z):=\bar{v}_{\varepsilon}\left(R-|z|+c_{\varepsilon}\right), \quad z \in T_{\rho}\left(\partial B_{R+c_{\varepsilon}}\right) & \text { in Case 2. }
\end{array}
$$

Thanks to (41) (or to (43)) we have $\lim _{\varepsilon \rightarrow 0^{+}} u_{\varepsilon}=\chi_{E \cap T_{\rho}\left(\partial B_{R}\right)}$ in $L_{\text {loc }}^{1}\left(T_{\rho}\left(\partial B_{R}\right)\right.$ ). Let $\bar{y}_{\varepsilon}:=\varepsilon^{-1} \delta_{\varepsilon}$ in Case 1 , and $\bar{y}_{\varepsilon}:=\varepsilon^{-1} c_{\varepsilon}$ in Case 2. Set $\widehat{I}_{\varepsilon}:=I_{\varepsilon}-\bar{y}_{\varepsilon}$ and let us define the function $U_{\varepsilon}: \widehat{I}_{\varepsilon} \rightarrow \mathbb{R}$ as follows:

$$
U_{\varepsilon}(y):=V_{\varepsilon}\left(y+\bar{y}_{\varepsilon}\right), \quad y \in \widehat{I}_{\varepsilon} .
$$




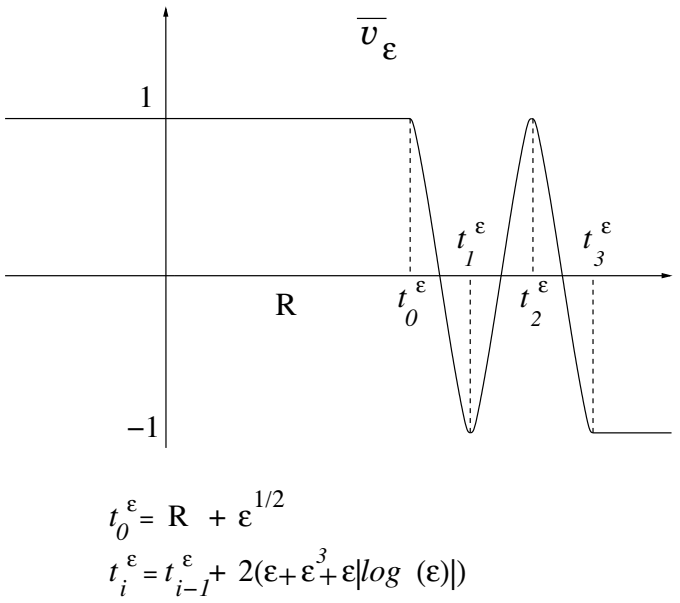

Fig. 2. Suppose that for $t \in] t_{i}^{\varepsilon}, t_{i+1}^{\varepsilon}\left[\right.$ and $i=0,1,2$ the function $\bar{v}_{\varepsilon}(t)$ is defined using a suitable translation of $\gamma_{\varepsilon}$, where $\gamma_{\varepsilon}$ is the optimal sequence for the $\Gamma$-lim sup inequality for $M_{\varepsilon}$; for instance, if $W(t)=\frac{\left(1-t^{2}\right)^{2}}{2}$, we can take $\gamma_{\varepsilon}$ to be the even and $\mathcal{C}^{1,1}$ function defined as $\gamma_{\varepsilon}(t):=\operatorname{tgh}(t / \varepsilon)$ if $0 \leq t<\varepsilon|\log \varepsilon|, \gamma_{\varepsilon}(t)$ equals a suitable arc of parabola if $\varepsilon|\log \varepsilon| \leq t \leq \varepsilon+\varepsilon^{3}+\varepsilon|\log \varepsilon|$, and $\gamma_{\varepsilon}(t)=+1$ if $t>\varepsilon+\varepsilon^{3}+\varepsilon|\log \varepsilon|$. For the sequence $\left\{v_{\varepsilon}\right\}$ (corresponding to $\left\{\bar{v}_{\varepsilon}\right\}$ ) we have: $v_{\varepsilon} \rightarrow \chi_{B_{R}}$ in $L_{\text {loc }}^{1}\left(\mathbb{R}^{2}\right) ; \mu_{\varepsilon}^{v_{\varepsilon}} \rightarrow \mu=3 c_{0} \mathcal{H}_{\mid \partial B_{R}}^{1}$. Moreover we have $\alpha \equiv 1$ and $\beta=\gamma$ (resp. $\beta=-\gamma$ ) if $t_{1}^{\varepsilon}-R \leq \delta_{\varepsilon} \leq t_{2}^{\varepsilon}-R$ (resp. $\left.t_{0}^{\varepsilon}-R \leq \delta_{\varepsilon} \leq t_{1}^{\varepsilon}-R\right)$

Observe that $U_{\varepsilon}=\bar{u}_{\varepsilon} \circ \phi_{\varepsilon}$. We have

$$
\bar{y}_{\varepsilon} \in I_{\varepsilon}, \quad V_{\varepsilon}\left(\bar{y}_{\varepsilon}\right)=0 \quad \forall \varepsilon>0
$$

and

$$
\lim _{\varepsilon \rightarrow 0^{+}} \varepsilon \bar{y}_{\varepsilon}=0
$$

By (57) it follows that $\frac{\rho / \varepsilon}{\bar{y}_{\varepsilon}} \rightarrow+\infty$ as $\varepsilon \rightarrow 0^{+}$, hence the intervals $\widehat{I}_{\varepsilon}$ invade $\mathbb{R}$ as $\varepsilon \rightarrow 0$. In addition

$$
\begin{array}{lrl}
U_{\varepsilon}(0)=0 \quad \text { for any } \varepsilon>0 & \text { in Case 1, } \\
U_{\varepsilon}(0)=1-\eta \quad \text { for any } \varepsilon>0 & \text { in Case 2. }
\end{array}
$$

Step 6. In Case 1 the sequence $\left\{U_{\varepsilon}\right\}$ converges to the minimizer $\gamma$ (defined in (15)) or to $-\gamma$ strongly in $H_{\text {loc }}^{2}(\mathbb{R})$. In Case 2 the sequence $\left\{U_{\varepsilon}\right\}$ converges to a translated of $\gamma$ or of $-\gamma$ strongly in $H_{\text {loc }}^{2}(\mathbb{R})$.

Suppose we are in Case 1. Arguing as in steps 3,4 and using (58), we deduce that there exists a function $\beta \in H_{\text {loc }}^{2}(\mathbb{R})$ such that $U_{\varepsilon} \rightarrow \beta$ weakly in $H_{\text {loc }}^{2}(\mathbb{R})$ as $\varepsilon \rightarrow 0^{+}, \beta(0)=0$, and

$$
\begin{aligned}
& \int_{\mathbb{R}}\left[\frac{\left(\beta^{\prime}\right)^{2}}{2}+W(\beta)\right] d y<+\infty, \\
& -\beta^{\prime \prime}(t)+W^{\prime}(\beta(t))=0, \quad \forall t \in \mathbb{R} .
\end{aligned}
$$


Hence $\beta$ equals either $\gamma$ or $-\gamma$.

Without loss of generality we can suppose $\beta=\gamma$. Set

$$
\sigma_{U_{\varepsilon}}:=-U_{\varepsilon}^{\prime \prime}+W^{\prime}\left(U_{\varepsilon}\right) .
$$

Observe that, from (54), we have

$$
\sup _{\varepsilon} \varepsilon^{-1}\left\|\sigma_{U_{\varepsilon}}\right\|_{L^{2}\left(\widehat{I}_{\varepsilon}\right)}<+\infty .
$$

In particular

$$
\lim _{\varepsilon \rightarrow 0^{+}}\left\|\sigma_{U_{\varepsilon}}\right\|_{L^{2}\left(\widehat{I}_{\varepsilon}\right)}=0 .
$$

Given a compact set $K \subset \mathbb{R}$, we then have

$$
\begin{aligned}
& \left\|U_{\varepsilon}^{\prime \prime}-\gamma^{\prime \prime}\right\|_{L^{2}(K)} \\
\leq & \left\|U_{\varepsilon}^{\prime \prime}-W^{\prime}\left(U_{\varepsilon}\right)\right\|_{L^{2}(K)}+\left\|W^{\prime}\left(U_{\varepsilon}\right)-W^{\prime}(\gamma)\right\|_{L^{2}(K)}+\left\|W^{\prime}(\gamma)-\gamma^{\prime \prime}\right\|_{L^{2}(\mathbb{R})} \\
= & \left\|\sigma_{U_{\varepsilon}}\right\|_{L^{2}(K)}+\left\|W^{\prime}\left(U_{\varepsilon}\right)-W^{\prime}(\gamma)\right\|_{L^{2}(K)} \rightarrow 0 \quad \text { as } \varepsilon \rightarrow 0^{+},
\end{aligned}
$$

where the last convergence follows from (62) and the uniform convergence of $U_{\varepsilon}$ to $\gamma$ on the compact subsets of $\mathbb{R}$. Hence

$$
U_{\varepsilon} \rightarrow \gamma \quad \text { strongly in } H_{\mathrm{loc}}^{2}(\mathbb{R}) \text { as } \varepsilon \rightarrow 0^{+},
$$

and step 6 is proved in Case 1.

In Case 2 we can repeat the proof of Case 1 using (45) instead of (58). Hence we end up with a function $\widehat{\beta} \in H_{\text {loc }}^{2}(\mathbb{R})$ such that $\widehat{\beta}(0)=1-\eta$ and such that (60) holds with $\widehat{\beta}$ in place of $\beta$. This is enough to conclude that $\widehat{\beta}(t)=\gamma(t+\nu)$ or that $\widehat{\beta}(t)=-\gamma(t+\nu)$, for some real number $\nu$.

We now conclude the proof of the theorem in Case 1. To obtain the proof in Case 2 it is enough to replace everywhere $\delta_{\varepsilon}$ with $c_{\varepsilon}$ and $\gamma(\cdot)$ with $\gamma(\cdot+\nu)$.

Step 7. We have

$$
\liminf _{\varepsilon \rightarrow 0^{+}} \varepsilon^{-1} \int_{T_{\varrho}\left(\partial B_{R}\right)}\left(\operatorname{eul}_{\varepsilon}\left(v_{\varepsilon}\right)\right)^{2} d z=\liminf _{\varepsilon \rightarrow 0^{+}} \varepsilon^{-1} \int_{T_{\varrho}\left(\partial B_{R+\delta_{\varepsilon}}\right)}\left(\operatorname{eul}_{\varepsilon}\left(u_{\varepsilon}\right)\right)^{2} d z
$$

In fact, using (34) and making the change of variable $\tau=y-\bar{y}_{\varepsilon}$ we get

$$
\begin{aligned}
& \varepsilon^{-1} \int_{T_{\varrho}\left(\partial B_{R}\right)}\left(\operatorname{eul}_{\varepsilon}\left(v_{\varepsilon}\right)\right)^{2} d z \\
= & 2 \pi R \int_{I_{\varepsilon}}\left[\varepsilon^{-1} \sigma_{V_{\varepsilon}}(y)+\frac{\kappa}{1-\kappa \varepsilon y} V_{\varepsilon}^{\prime}(y)\right]^{2}|1-\kappa \varepsilon y| d y \\
= & 2 \pi R \int_{I_{\varepsilon}-\bar{y}_{\varepsilon}}\left[\varepsilon^{-1} \sigma_{V_{\varepsilon}}\left(\tau+\bar{y}_{\varepsilon}\right)+\frac{\kappa}{1-\kappa \varepsilon\left(\tau+\bar{y}_{\varepsilon}\right)} V_{\varepsilon}^{\prime}\left(\tau+\bar{y}_{\varepsilon}\right)\right]^{2}\left|1-\kappa \varepsilon\left(\tau+\bar{y}_{\varepsilon}\right)\right| d \tau \\
= & 2 \pi R \int_{\widehat{I}_{\varepsilon}}\left[\varepsilon^{-1} \sigma_{U_{\varepsilon}}(\tau)+\frac{\kappa}{1-\kappa \varepsilon \tau-\kappa \varepsilon \bar{y}_{\varepsilon}} U_{\varepsilon}^{\prime}(\tau)\right]^{2}\left|1-\kappa \varepsilon \tau-\kappa \varepsilon \bar{y}_{\varepsilon}\right| d \tau .
\end{aligned}
$$


Similarly

$$
\begin{aligned}
& \varepsilon^{-1} \int_{T_{\varrho}\left(\partial B_{R+\delta_{\varepsilon}}\right)}\left(\operatorname{eul}_{\varepsilon}\left(u_{\varepsilon}\right)\right)^{2} d z \\
= & 2 \pi R \int_{\widehat{I}_{\varepsilon}}\left[\varepsilon^{-1} \sigma_{U_{\varepsilon}}(\tau)+\frac{\kappa}{1-\kappa \varepsilon \tau} U_{\varepsilon}^{\prime}(\tau)\right]^{2}|1-\kappa \varepsilon \tau| d \tau .
\end{aligned}
$$

By (57) and the analogue of (48) and (53) for $U_{\varepsilon}$, we have

$$
\begin{aligned}
& \int_{\widehat{I}_{\varepsilon}}\left[\varepsilon^{-1} \sigma_{U_{\varepsilon}}(\tau)+\frac{\kappa}{1-\kappa \varepsilon \tau-\kappa \varepsilon \bar{y}_{\varepsilon}} U_{\varepsilon}^{\prime}\right]^{2}\left|1-\kappa \varepsilon \tau-\kappa \varepsilon \bar{y}_{\varepsilon}\right| d \tau \\
= & \int_{\widehat{I}_{\varepsilon}}\left[\varepsilon^{-1} \sigma_{U_{\varepsilon}}(\tau)+\frac{\kappa}{1-\kappa \varepsilon \tau} U_{\varepsilon}^{\prime}(\tau)\right]^{2}|1-\kappa \varepsilon \tau| d \tau+o(1),
\end{aligned}
$$

where $\lim _{\varepsilon \rightarrow 0^{+}} O(1)=0$. Hence (64) follows.

Step 8. Conclusion of the proof of (32).

In view of (64), we have to show that

$$
\frac{2 \pi c_{0}}{R}=c_{0} \int_{\partial B_{R}}(\kappa(z))^{2} d \mathcal{H}^{1}(z) \leq \liminf _{\varepsilon \rightarrow 0^{+}} \varepsilon^{-1} \int_{T_{\varrho}\left(\partial B_{R+\varepsilon \bar{y}_{\varepsilon}}\right)}\left(\operatorname{eul}_{\varepsilon}\left(u_{\varepsilon}\right)\right)^{2} d z .
$$

Using the analogue of (37) for $U_{\varepsilon}$ and $\kappa=1 / R$, this is equivalent to show that

$$
\frac{2 \pi}{R} \int_{\mathbb{R}}\left|\gamma^{\prime}\right|^{2} d y \leq 2 \pi R \liminf _{\varepsilon \rightarrow 0^{+}} \int_{\widehat{I}_{\varepsilon}}\left[\varepsilon^{-1} \sigma_{U_{\varepsilon}}+\frac{\kappa}{1-\kappa \varepsilon y} U_{\varepsilon}^{\prime}\right]^{2}|1-\kappa \varepsilon y| d y .
$$

Since

$$
\left[\varepsilon^{-1} \sigma_{U_{\varepsilon}}+\frac{\kappa}{1-\kappa \varepsilon y} U_{\varepsilon}^{\prime}\right]^{2} \geq \frac{\kappa^{2}}{(1-\kappa \varepsilon y)^{2}}\left(U_{\varepsilon}^{\prime}\right)^{2}+2 \frac{\varepsilon^{-1} \kappa \sigma_{U_{\varepsilon}}}{1-\kappa \varepsilon y} U_{\varepsilon}^{\prime},
$$

recalling (30), inequality (65) is proved if we show that

$$
\frac{1}{R} \int_{\mathbb{R}}\left|\gamma^{\prime}\right|^{2} d y \leq R \liminf _{\varepsilon \rightarrow 0^{+}} \int_{\widehat{I}_{\varepsilon}}\left(\frac{\kappa^{2}}{1-\kappa \varepsilon y}\left(U_{\varepsilon}^{\prime}\right)^{2}+2 \varepsilon^{-1} \kappa \sigma_{U_{\varepsilon}} U_{\varepsilon}^{\prime}\right) d y .
$$

Denoting by

$$
\begin{aligned}
& \mathrm{A}_{\varepsilon}:=R \int_{\widehat{I_{\varepsilon}}} \frac{\kappa^{2}}{1-\kappa \varepsilon y}\left(U_{\varepsilon}^{\prime}\right)^{2} d y=\frac{1}{R} \int_{\widehat{I_{\varepsilon}}} \frac{1}{1-\kappa \varepsilon y}\left(U_{\varepsilon}^{\prime}\right)^{2} d y, \\
& \mathrm{~B}_{\varepsilon}:=2 R \int_{\widehat{I}_{\varepsilon}} \varepsilon^{-1} \kappa \sigma_{U_{\varepsilon}} U_{\varepsilon}^{\prime} d y=2 \varepsilon^{-1} \int_{\widehat{I}_{\varepsilon}} \sigma_{U_{\varepsilon}} U_{\varepsilon}^{\prime} d y,
\end{aligned}
$$

we must show that

$$
\frac{1}{R} \int_{\mathbb{R}}\left|\gamma^{\prime}\right|^{2} d y \leq \liminf _{\varepsilon \rightarrow 0^{+}} \mathrm{A}_{\varepsilon}+\liminf _{\varepsilon \rightarrow 0^{+}} \mathrm{B}_{\varepsilon}
$$


We are going to prove that

$$
\lim _{\varepsilon \rightarrow 0^{+}} \mathrm{B}_{\varepsilon}=0
$$

Note carefully that it is exactly in the effort of proving (66) that (28) is required.

Integrating by parts and using the relation $V_{\varepsilon}\left( \pm \varepsilon^{-1} \rho\right)=\bar{v}_{\varepsilon}( \pm \rho)$ we have

$$
\begin{aligned}
\frac{\mathrm{B}_{\varepsilon}}{2} & =\varepsilon^{-1} \int_{\widehat{I}_{\varepsilon}} U_{\varepsilon}^{\prime}\left(-U_{\varepsilon}^{\prime \prime}+W^{\prime}\left(U_{\varepsilon}\right)\right) d y=\varepsilon^{-1} \int_{\widehat{I}_{\varepsilon}}\left(\frac{-\left(U_{\varepsilon}^{\prime}\right)^{2}}{2}+W\left(U_{\varepsilon}\right)\right)^{\prime} d y \\
& =\varepsilon^{-1}\left[-\frac{\left(V_{\varepsilon}^{\prime}\left(\varepsilon^{-1} \rho\right)\right)^{2}}{2}+W\left(V_{\varepsilon}\left(\varepsilon^{-1} \rho\right)\right)+\frac{\left(V_{\varepsilon}^{\prime}\left(-\varepsilon^{-1} \rho\right)\right)^{2}}{2}-W\left(V_{\varepsilon}\left(-\varepsilon^{-1} \rho\right)\right)\right] \\
& =\left(-\frac{\varepsilon}{2}\left(\bar{v}_{\varepsilon}^{\prime}(\rho)\right)^{2}+\varepsilon^{-1} W\left(\bar{v}_{\varepsilon}(\rho)\right)\right)+\left(\frac{\varepsilon}{2}\left(\bar{v}_{\varepsilon}^{\prime}(-\rho)\right)^{2}-\varepsilon^{-1} W\left(\bar{v}_{\varepsilon}(-\rho)\right)\right) .
\end{aligned}
$$

Then (66) follows by using (28).

Furthermore,

$$
\mathrm{A}_{\varepsilon} \geq \frac{1}{R} \int_{\widehat{I}_{\varepsilon}}\left(U_{\varepsilon}^{\prime}\right)^{2} d y
$$

Hence, given a compact set $K \subset \mathbb{R}$, from Fatou's lemma we have

$$
\liminf _{\varepsilon \rightarrow 0^{+}} \mathrm{A}_{\varepsilon} \geq \frac{1}{R} \lim _{\varepsilon \rightarrow 0^{+}} \int_{K}\left(U_{\varepsilon}^{\prime}\right)^{2} d y \geq \frac{1}{R} \int_{K}\left|\gamma^{\prime}\right|^{2} d y
$$

Hence, passing to the supremum with respect to $K$, we get $\liminf _{\varepsilon \rightarrow 0^{+}} \mathrm{A}_{\varepsilon} \geq$ $\frac{1}{R} \int_{\mathbb{R}}\left|\gamma^{\prime}\right|^{2} d y$. This concludes the proof.

Conclusion of the proof of Theorem 1.1.

As a direct consequence of Theorem 4.1 and Remark 3.4 we obtain (6).

By Corollary 3.5 we know that $\operatorname{spt}(\mu)$ consists of a family of circles $\left\{\partial B_{R_{i}}\right\}_{i \in I}$, where $I$ is at most countable, and these circles may accumulate only at the origin. Without loss of generality, we can assume that $i<j$ implies $R_{i}<R_{j}$. Thanks to Theorem 4.1, for any $i \in I$ we can choose $\rho_{i}>0$ in such a way that $R_{i+1}<$ $R_{i}-\rho_{i}<R_{i}+\rho_{i}<R_{i-1}$ and

$$
\liminf _{\varepsilon \rightarrow 0^{+}} \mathcal{F}_{\varepsilon}\left(v_{\varepsilon}, T_{\rho_{i}}\left(\partial B_{R_{i}}\right)\right) \geq 2 \pi c_{0}\left(R_{i}+\frac{1}{R_{i}}\right) .
$$

We then have

$$
\begin{aligned}
\liminf _{\varepsilon \rightarrow 0^{+}} \mathcal{F}_{\varepsilon}\left(v_{\varepsilon}\right) & \geq \sum_{i \in I} \liminf _{\varepsilon \rightarrow 0^{+}} \mathcal{F}_{\varepsilon}\left(v_{\varepsilon}, T_{\rho_{i}}\left(\partial B_{R_{i}}\right)\right) \\
& \geq 2 \pi c_{0} \sum_{i \in I}\left(R_{i}+\frac{1}{R_{i}}\right) .
\end{aligned}
$$

By (5) it follows that $\sum_{i \in I}\left(R_{i}+\frac{1}{R_{i}}\right)<+\infty$, and this implies that $I$ is a finite set. This concludes the proof of Theorem 1.1. 
Remark 4.2. From the proof of Theorem 4.1 and using Theorem 1.1 we can prove a stronger property of the limit measure $\mu$, namely

$$
\begin{aligned}
& \lim _{r \rightarrow 0^{+}} \frac{\mu\left(B_{r}\left(z_{0}\right)\right)}{r}=2 m c_{0}, \quad \text { if } z_{0} \in \operatorname{spt}(\mu) \cap\left(\mathbb{R}^{2} \backslash \partial E\right) \\
& \lim _{r \rightarrow 0^{+}} \frac{\mu\left(B_{r}\left(z_{0}\right)\right)}{r}=(2 m+1) c_{0}, \quad \text { if } z_{0} \in \operatorname{spt}(\mu) \cap \partial E,
\end{aligned}
$$

for some positive integer number $m$ depending on $\left|z_{0}\right|$. Indeed, from (24) and the strong- $H_{\text {loc }}^{2}(\mathbb{R})$ convergence to $\pm \gamma$ of the sequence $\left\{U_{\varepsilon}\right\}$ defined in step 5 , we obtain that (at least on a subsequence) the set $\left\{y \in \widehat{I}_{\varepsilon}: U_{\varepsilon}(y)=0\right\}$ is finite. Then (68) follows from the continuity of $v_{\varepsilon}$ and the convergence of $\left\{v_{\varepsilon}\right\}$ to $\chi_{E}$ in $L_{\text {loc }}^{1}\left(\mathbb{R}^{2}\right)$.

\section{Final Comments}

(1) With minor modifications, inequality (6) in Theorem 1.1 holds in any space dimension $n$, i.e., replacing $\mathbb{R}^{2}$ with $\mathbb{R}^{n}$ in the statement (where $\kappa$ in the definition (4) of the functional $F$ stands for the sum of the principal curvatures $\kappa_{i}$ of $\partial E$ positive for the sphere, and where (11) must be replaced by $\left.\sum_{i=1}^{n-1} \frac{\kappa_{i}(\pi(z))}{1-d(z) \kappa_{i}(\pi(z))}=-\Delta d(z)\right)$.

(2) In dimension $n>3$, the assertion concerning $\operatorname{spt}(\mu)$ in Theorem 1.1 is not anymore true. Indeed (see (67)), assuming

$$
\sum_{i \in I}\left(R_{i}^{n-1}+(n-1)^{2} R_{i}^{n-3}\right)<+\infty
$$

does not imply that $\operatorname{spt}(\mu)$ consists of a finite number of spheres. On the other hand, the assertion is still valid in $n=3$ dimensions.

(3) With minor modification, inequality (6) is valid (in any dimension $n$ ) for a sequence $\left\{v_{\varepsilon}\right\}$ converging in $L_{\mathrm{loc}}^{1}\left(\mathbb{R}^{n}\right)$ to an open set $E$ with smooth compact boundary, assuming that each $v_{\varepsilon}$ depends only on the distance from $\partial E$.

(4) Recall that, given an open set $E \subset \mathbb{R}^{n}$ with compact boundary of class $\mathcal{C}^{2}$, there exists a sequence $\left\{v_{\varepsilon}\right\} \subset H_{\text {loc }}^{2}\left(\mathbb{R}^{n}\right)$ of functions converging to $\chi_{E}$ in $L^{1}\left(\mathbb{R}^{n}\right)$ and such that $c_{0} F(E)=\lim _{\varepsilon \rightarrow 0^{+}} \mathcal{F}_{\varepsilon}\left(v_{\varepsilon}\right)$, see [5].

\section{References}

1. Alberti, G.: Variational models for phase transitions, an approach via $\Gamma$-convergence. In: Buttazzo, G., Marino, A., Murthy M.K.V. (eds.) Calculus of variations and partial differential equations, topics on geometrical evolutions problems and degree theory, pp. 95-114. Springer, Berlin Heidelberg New York 1998

2. Ambrosio, L., Fusco, N., Pallara, D.: Functions of bounded variation and free discontinuity problems. Oxford Mathematical Monographs. Clarendon Press, Oxford 2000

3. Bellettini, G., Dal Maso, G., Paolini, M.: Semicontinuity and relaxation properties of a curvature depending functional in 2D. Ann. Scuola Norm. Sup. Pisa Cl. Sci. (4) 20, 247-297 (1993)

4. Bellettini, G., Mugnai, L.: Characterization and representation of the lower semicontinuous envelope of the elastica functional. Ann. Inst. H. Poincaré, Anal. Non Linéaire 21, 839-880 (2004) 
5. Bellettini, G., Paolini, M.: Approssimazione variazionale di funzionali con curvatura. Seminario di Analisi Matematica Univ. Bologna, Tecnoprint, pp. 87-97. Bologna (1993)

6. Chen, X.: Global asymptotic limit of solutions of the Cahn-Hilliard equation. J. Differential Geom. 44, 262-311 (1996)

7. Dal Maso, G., Modica, L.: A general theory of variational functionals. Topics in Funtional Analysis, 1980-1981, pp. 149-221. Quaderni, Scuola Normale Superiore di Pisa (1981)

8. De Giorgi, E.: Some remarks on $\Gamma$-convergence and least squares method. In: Dal Maso, G., Dell'Antonio, G.F. (eds.) Composite media and homogeneization theory, pp. 135-142. Birkhäuser, Boston 1990

9. De Giorgi, E., Franzoni, T.: Su un tipo di convergenza variazionale. Atti Accad. Naz. Lincei, Cl. Sci. Fis. Mat. Nat. 58, 842-850 (1975)

10. Giusti, E.: Minimal surfaces and functions of bounded variation. Birkhäuser, Boston 1984

11. Hutchinson, J.E., Tonegawa, Y.: Convergence of phase interfaces in the van der WaalsCahn-Hilliard theory. Calc. Var. Partial Differential Equations 10, 49-84 (2000)

12. Ilmanen, T.: Convergence of the Allen-Chan equation to Brakke's motion by mean curvature. J. Differential Geom. 38, 417-461 (1993)

13. Modica, L.: Gradient theory of phase transitions and minimal interface criterion. Arch. Rational Mech. Anal. 98, 123-142 (1987)

14. Modica, L., Mortola, S.: Un esempio di $\Gamma$-convergenza. Boll. Un. Mat. Ital. (5) 14 B, 285-299 (1977)

15. Padilla, P., Tonegawa, Y.: On the convergence of stable phase transitions. Comm. Pure Appl. Math. 51, 551-579 (1978)

16. Tonegawa, Y.: Phase field model with a variable chemical potential. Proc. Royal Soc. Edinburgh 132 A, 993-1019 (2002) 